\title{
TT Arietis - observations of a Cataclysmic Variable Star with the MOST Space Telescope
}

\author{
J. Weingrill, ${ }^{1}$ G. Kleinschuster, ${ }^{2}$ R. Kuschnig, ${ }^{3}$ J. M. Matthews, ${ }^{4}$ A. Moffat, ${ }^{5}$ S. Rucinski, ${ }^{6}$ \\ D. Sasselov, ${ }^{7}$ and W. W. Weiss ${ }^{3}$ \\ ${ }^{1}$ Space Research Institute, Austrian Academy of Sciences, Schmiedlstrasse 6, 8042 Graz, Austria \\ 2 Astro Club Auersbach, Wetzelsdorf 33, 8330 Feldbach, Austria \\ 3 Institut für Astronomie, Universität Wien, Türkenschanzstrasse 17, 1180 Wien, Austria \\ ${ }^{4}$ Department of Physics \& Astronomy, University of British Columbia, 6224 Agricultural Road, \\ Vancouver, BC V6T 1Z1, Canada \\ ${ }^{5}$ Departément de physique, Université de Montréal C.P. 6128, Succ. Centre-Ville, \\ Montréal, QC H3C 3J7, Canada \\ ${ }^{6}$ David Dunlap Observatory, Department of Astronomy, University of Toronto P.O. Box 360, \\ Richmond Hill, ON L4C 4Y6, Canada \\ 7 Harvard-Smithsonian Center for Astrophysics, 60 Garden Street, \\ Cambridge, Massachusetts, MA 02138, USA
}

\begin{abstract}
We measured the photometric flux of the cataclysmic variable TT Arietis (BD+14 341) using the MOST space telescope. Periodic oscillations of the flux reveal the photometric period as well as other features of this binary system. We applied a Discrete Fourier Transform (DFT) on a reduced data set to retrieve the frequencies of TT Arietis. The analysis of the system revealed a primary photometric period of 3.19 hours. Though the MOST data has a high cadence of 52.8 seconds, a fine structure of the accretion disk is not obvious.
\end{abstract}

Individual Objects: TT Ari

\section{Introduction}

The MOST (Microvariability and Oscillations of STars) satellite observed TT Ari between MJD 54395.6 and 54406.4. The optical setup of MOST consists of a Maksutov type optical telescope and two identical CCDs. A detailed description of the MOST mission can be found in Walker et al. (2003) and Matthews (2004). The classification of the star is hindered by the low inclination of 20 degrees and therefore mentioned differently in literature. It is most likely to be a VY Scl-type star (Wu et al. 2002) or belongs to the class of SW Sex stars as mentioned by Kim et al. (2009). The primary photometric period varies between 3.1824(48) hours and 3.19056(72) hours as listed in Tremko et al. (1996). According to the observations of quasi-periodic oscillations (QPO) between 2005 and 2006, TT Ari is believed to return from its 'positive superhump' state (Kim et al. 2009).

\section{Methods}

The target was measured with direct imaging photometry because of the low magnitude of the TT Ari system. The initial data reduction has been accomplished by the MOST science 


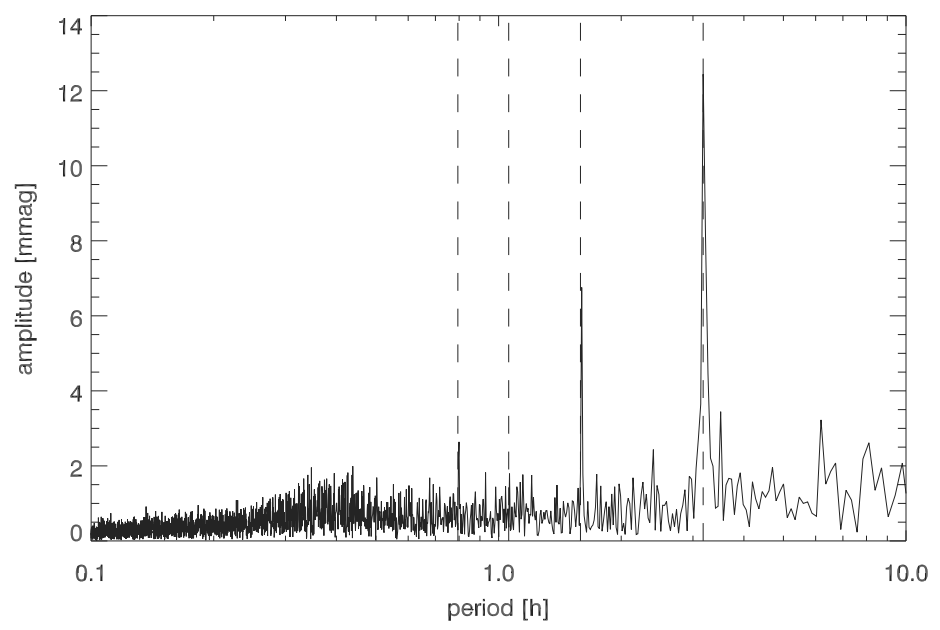

Figure 1: Periodogram derived from the DFT analysis. The main period and its harmonics are indicated by dashed lines.

team (Rowe et al. 2006). The first analysis of the data was carried out using discrete Fourier transform (Swan 1982). The Fourier autocorrelation function (Scargle 1989) was used as an alternate method, since it shows 36 periods which are not obvious in the original data. In order to remove the remaining signature of the satellite orbit, the first 100 data points equivalent to a time lag of 1.452 hours were ignored. The distances between the first three local maxima were averaged to obtain the period from the autocorrelation function. In order to look for QPOs and variations in the periodicity of the signal indicated by the autocorrelation function, a sliding Fourier window analysis (Jacobsen \& Lyons 2003) was carried out. The Fourier window sizes were scaled from 1.88 hours to 15 hours and a boxcar function was used as a windowing function. The window was shifted over the data points without overlapping.

\section{Results}

The analysis using DFT reveals a period of 3.177(44) hours with an amplitude of $12.4 \mathrm{mmag}$. The resulting periodogram can be seen in Fig. 1 . The main period and the amplitudes of its harmonics correspond to a nearly sinusoidal shape of the folded light curve. Other methods like the autocorrelation of the data show a slightly different photometric period of 3.194(18) hours.

The QPOs which can be identified as flickering of the accretion disk is visible in the regime of 19 to 26 minutes. This corresponds to earlier results (see e.g. Semeniuk et al., 1987) in a 'negative superhump' state. Due to the short observation run of the MOST satellite the current 'superhump state' could not be verified.

Acknowledgments. Data was provided by 'Universe in a Suitcase - MOST for all'.

\section{References}

Walker, G., Matthews, J. M., Kuschnig, R., et al. 2003, PASP, 115, 1023

Matthews, J. M., 2004, Bullet. of the AAS, 35, 1563 
Wu, X., Li, Z., Ding, Y., et al. 2002, ApJ, 569, 418

Kim, Y., Andronov, I. L., Cha, S. M., et al. 2009, A\&A, in press, arXiv:0810.1489

Tremko, J., Andronov, I. L., Chinarova, L. L., et al. 1996, A\&A, 312, 121

Rowe, J. F., Matthews, J. M., Seager, S., et al. 2006, ApJ, 646, 1241

Swan, R. R. 1982, AJ, 87, 1608

Scargle, J. D. 1989, ApJ, 343, 874

Jacobsen, E., \& Lyons, R. 2003, SP-M, 74

Semeniuk I., Schwarzenberg-Czerny A., Duerbeck H., et al. 1987, A\&A, 37, 197
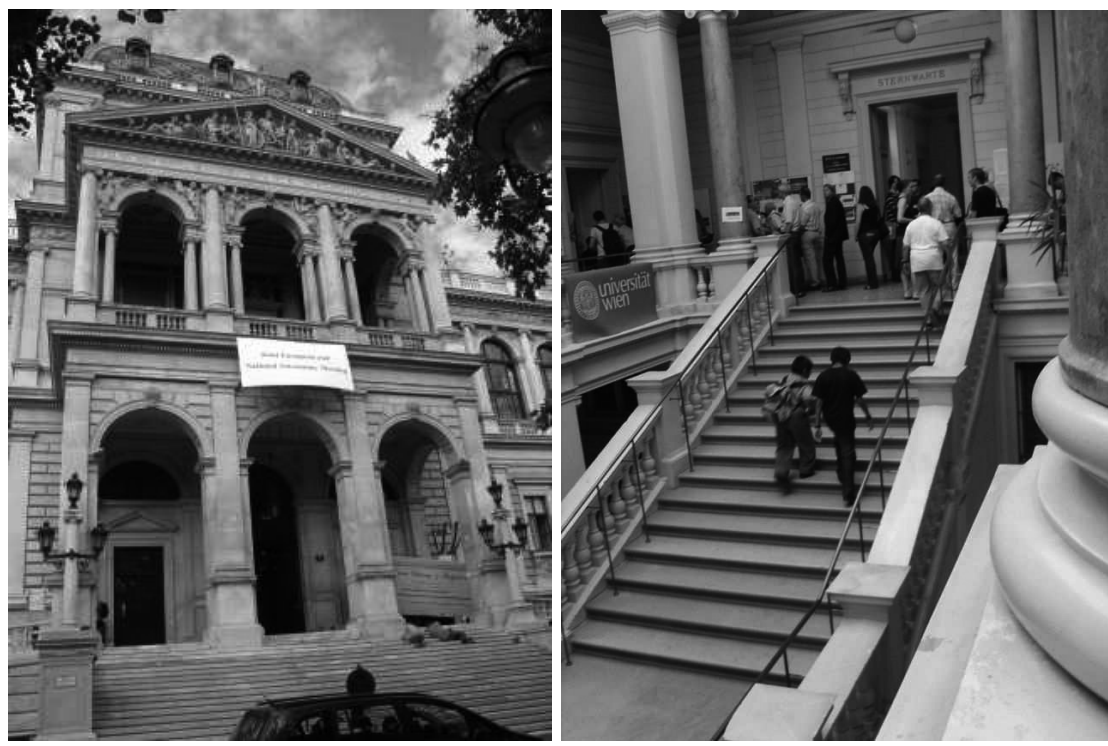

The conference venue: main building of the University of Vienna; and reception and registration at the University Observatory. 